\title{
25 Multiphysics modeling of porous ferrogels at finite strains
}

\begin{abstract}
Porous ferrogels are a new class of magnetoactive composite materials that consist of a polymeric hydrogel matrix with embedded magnetizable particles. The mutual particle interaction within the soft elastic matrix enables ferrogels to deform and alter their material characteristics upon magnetic stimulation. Due to these unique properties, ferrogels have attracted significant attention for potential uses in a variety of engineering applications, especially in biomedical engineering and microfluidics. Therefore, it is crucial to develop precise mathematical models capturing the complex material behavior of ferrogels, which spans over multiple length scales. The aim of this work is to present suitable modeling approaches for porous ferrogels. Following the hierarchical structure of scales, we present modeling frameworks for two different scenarios: (i) the modeling of ferrogels at the macroscale level and (ii) the modeling of ferrogels at the microscale level. Regarding the constitutive modeling of ferrogels, we limit our attention to locally nondissipative isotropic material response. For both modeling approaches, we provide comprehensive variational principles and briefly discuss relevant ingredients of a stable finite element implementation. In each section, numerical simulations are outlined in order to demonstrate the capabilities and relevant features of each modeling approach. Main emphasis of the numerical studies lies on the investigation of the macroscopic shape effect as well as on the characterization of the magnetomechanical material response of ferrogels with random monodisperse microstructures.
\end{abstract}

Keywords: computational homogenization, finite element analysis, large deformations, magnetoactive polymers, magnetomechanics, magneto-poro-mechanics, porous ferrogels

\subsection{Introduction}

In recent years, there has been a growing interest in the study of the behavior of fieldresponsive or so-called smart polymers. Porous ferrogels are a special class of these

\footnotetext{
*Corresponding author: Thomas Wallmersperger, Institute of Solid Mechanics, TU Dresden, Dresden, Germany, E-mail: thomas.wallmersperger@tu-dresden.de

Philipp Gebhart and Abdolhamid Attaran, Institute of Solid Mechanics, TU Dresden, Dresden, Germany, E-mail: philipp.gebhart@tu-dresden.de (P. Gebhart), hamid.attaran@gmail.com (A. Attaran)

Dopen Access. @ 2020 Philipp Gebhart et al., published by De Gruyter. @) BY-NC-ND This work is licensed under the Creative Commons Attribution-NonCommercial-NoDerivatives 4.0 International License.

This article has previously been published in the journal Physical Sciences Reviews. Please cite as: P. Gebhart, A. Attaran, and T. Wallmersperger "Multiphysics modeling of porous ferrogels at finite strains" Physical Sciences Reviews [Online] 2020, 5. DOI: $10.1515 /$ psr-2019-0091 | https://doi.org/10.1515/9783110569636-025
} 
materials consisting of a porous polymeric hydrogel matrix with dispersed microsized or nanosized magnetizable particles. The magneto-poro-mechanical behavior of these materials is a complex phenomenon that spans over multiple length scales and essentially depends on (i) the constitutive behavior of the individual components, (ii) their morphology and microstructural arrangement as well as (iii) the macroscopic geometry of the specimen. Typically, multidomain ferromagnetic particles with soft magnetic material characteristics are used as filler particles resulting in reversible fieldinduced deformation mechanisms. Based on the arrangement of the magnetic particles within the polymer matrix, ferrogels can exhibit isotropic or anisotropic material behavior on the macroscale. In case of a random distribution of the particles within the polymer matrix, which can be realized by the synthesis of the ferrogel in the absence of a magnetic field, the macroscopic material response will be isotropic. If the ferrogel is instead synthesized in the presence of a magnetic field, the magnetizable particles tend to arrange themselves in chain-like structures leading to a directional anistropy of the material on the macroscale. Due to their broad range of synthetically fabricable properties as well as their ability to exhibit large deformations and alter their effective material behavior upon external magnetic stimulation, ferrogels have received considerable attention in different branches of engineering in recent times. Possible applications in biomedical engineering comprise on-demand drug and cell delivery, see e.g. the studies by $\mathrm{Hu}$ et al. [1], Zhao et al. [2] and Cezar et al. [3], or regeneration of damaged biological tissues, see e.g. the studies by Li et al. [4] and Culver et al. [5]. An overview of further potential applications in biomedical engineering is given in the studies by Li et al. [4] and Datta [6]. Moreover, ferrogels have potential to be applied in microfluidics as actuators for active flow control, see the study by Hong et al. [7]. Due to their increasingly gaining importance in engineering, mathematical modeling of ferrogels has been an area of active research in the last few years.

In general, one can distinguish between microscopic modeling approaches, where the heterogeneous microstructure of the composite material is explicitly resolved, and macroscopic modeling approaches, where - under the assumption of scale separation - the composite is considered as a macroscopical homogenous continuum.

Particle interaction models at the microscale level have been proposed by Weeber et al. [8], Tarama et al. [9], Cremer et al. [10] and Fischer and Menzel [11], among others. These modeling approaches are based on the minimization of potentials, where the magnetic interaction between the particles is described via mutually interacting magnetic dipoles. Note that these dipole models are only suitable for the modeling of dilute systems with relatively low particle volume fractions, while at higher particle volume fractions, the assumption of magnetic dipoles is not valid anymore. In order to extend the applicability of these models to systems with higher volumetric particle concentrations, a multipole expansion can be used, see e.g. the study by Biller et al. [12, 13]. Furthermore, appropriate scale transition techniques have been presented by Menzel [14] and Pessot et al. [15] in order to bridge between the microscale and macroscale. 
Continuum-based modeling approaches for magnetoactive polymers at the microscale level were proposed by Galipeau and Ponte Castañeda [16, 17], Javili et al. [18], Kalina et al. [19, 20], Danas [21] and Metsch et al. [22, 23], among others. The fundamentals in the mathematical modeling of field-matter interaction in deformable continua were developed by Landau et al [24], Truesdell and Toupin [25], Brown [26], Tiersten [27], Coleman and Dill [28], de Groot and Suttorp [29], Maugin and Eringen [30], Eringen and Maugin [31], Pao [32], Hutter et al. [33] and Kovetz [34], among others. In contrast to the particle interaction models, the magnetic and mechanical fields are fully resolved within the continuum models. Therefore, continuum-based modeling approaches are not a priori restricted to the modeling of dilute systems with low particle volume fractions. These microscale models are usually embedded into an appropriate scale transition scheme as developed by Chatzigeorgiou et al. [35] in order to predict the effective material behavior of the composite at the macroscale. However, please note that these frameworks are limited to analyze the material behavior of heterogeneous microstructure at a single material point of the macrostructure. In order to account for macrostructural effects, $\mathrm{FE}^{2}$-techniques were developed by Keip and Rambausek [36, 37] and Rambausek et al. [38], where at each quadrature point of the macroscopic body, a microscopic boundary value problem with a microscopically representative volume element is solved.

The continuum modeling of ferrogels at the macroscale level has been addressed in the recent works of Nedjar [39], Attaran et al. [40, 41] and Gebhart and Wallmersperger [42]. These models have in common that the ferrogel is considered as a magnetoactive solid-fluid mixture on the macroscale. However, the parametrization of phenomenological constitutive models for magnetoactive polymers based on experiments poses some major challenges due to the crucial macrostructural geometry influence of the specimen as extensively discussed in the study by Keip and Rambausek [36].

The present work focuses on the multiscale continuum-based modeling of porous ferrogels. We present theoretical and computational frameworks for the modeling of ferrogels at the macroscale and microscale level. The field-matter interaction in both models is described based on the statistical model by de Groot and Suttorp [29] in the nonrelativistic approximation. For a comprehensive overview on the field-matter interaction in deformable continua, we refer to the studies by Maugin and Eringen [30], Eringen and Maugin [31], Pao [32], Hutter et al. [33], Kovetz [34], Kankanala and Triantafyllidis [43], Ogden and Steigmann [44], among others.

The work is organized as follows: Section 25.2 covers the macroscale modeling of porous ferrogels. In sections 25.2 .1 and 25.2.2, kinematics, field equations and modeling assumptions are outlined within a consistent thermodynamical framework. A phenomenological constitutive model for isotropic porous ferrogels in an enthalpy-based setting is developed in section 25.2.3. Section 25.2.4 introduces an incremental variational principle that governs the solution of the saddlepoint problem. In section 25.2.5, 
the modeling framework is applied to investigate the macroscopic shape effect for initially spheroidal shaped specimens.

Section 25.3 covers the microscale modeling of ferrogels. To this end, Section 25.3.1 and 25.3.2 first summarize kinematics and field equations for the quasi-static case of finite magnetomechanics in a Lagrangian geometric setting. Constitutive models for the polymeric gel matrix and the soft magnetizable particles in an energetic constitutive arrangement are presented in section 25.3.3. A suitable energybased scale transition scheme is introduced in section 25.3.4 in order to bridge between the microscale and the macroscale. A minimization principle for the solution of the boundary value problem and details of the finite element implementation are discussed in section 25.3.5. The developed framework is applied in section 25.3.6 to investigate the effective material response of ferrogels with random monodisperse particle distributions.

Section 25.4 closes the paper with some concluding remarks as well as an outlook to possible future work.

\subsection{Macroscale modeling of ferrogels}

In this chapter, we propose a framework for the macroscopic modeling of porous ferrogels at finite strains. We assume separation of the length scales of macroscale and microscale such that the ferrogel is treated as a multiphasic mixture on the macroscale consisting of magnetoactive solid phase $(s)$, a fluid phase $(f)$ and additional mobile particles $(m)$. Within this theory each spatial point is simultaneously occupied by all constituents. For an extensive review of mechanics of multiphase continua, we refer to Biot [45, 46], Lewis and Schrefler [47] and Coussy $[48,49]$, among others.

Notation: At this point, we want to introduce some basic symbolic notations in order to facilitate the readability of the text in the following chapters. Throughout the text scalar quantities are denoted by nonbold symbols, while vectors and higher-order tensors are indicated by bold symbols. The spatial gradient of a field is defined by $\nabla_{\boldsymbol{x}}(\cdot)$, its spatial divergence as $\operatorname{div}(\cdot)$ and its spatial rotation as curl (.). In analogy $\nabla_{\boldsymbol{X}^{\alpha}}(\cdot)$, $\operatorname{Div}_{\alpha}(\cdot)$ and $\operatorname{Curl}_{\alpha}(\cdot)$ denote these operations with respect to the reference coordinates of component $\alpha$.

\subsubsection{Kinematics}

Consider a multiphase mixture $B$ consisting of $\alpha=\{s, f, m\}$ components, where each body $B^{\alpha}$ consists of a fixed set of material particles $P^{\alpha} \in B^{\alpha}$ embedded in the Euclidean space at time $t \in \mathbb{R}^{+}$. Let their reference configurations be denoted by $\mathcal{B}^{\alpha} \subset \mathbb{R}^{3}$ and the current configuration by $\mathcal{S} \subset \mathbb{R}^{3}$. The corresponding referential and current positions of 
a material point $P^{\alpha}$ in the Euclidian space are given by $\boldsymbol{X}^{\alpha} \in \mathcal{B}^{\alpha}$ and $\boldsymbol{x} \in \mathcal{S}$, respectively. The motion of the material body $B^{\alpha}$ is a time-dependent family of configurations described by the bijective deformation map as follows:

$$
\boldsymbol{\varphi}^{\alpha}:=\left\{\begin{array}{l}
\mathcal{B}^{\alpha} \times T \rightarrow \mathcal{S} \subset \mathbb{R}^{3} \\
\left(\boldsymbol{X}^{\alpha}, t\right) \mapsto \boldsymbol{X}=\boldsymbol{\varphi}^{\alpha}\left(\boldsymbol{X}^{\alpha}, t\right)
\end{array}\right.
$$

which maps the referential position $\boldsymbol{X}^{\alpha} \in \mathcal{B}^{\alpha}$ of a particle $P^{\alpha}$ of component $\alpha$ to its current position $\boldsymbol{x} \in \mathcal{S}$ at time $t \in T \subset \mathbb{R}^{+}$. The material velocity and acceleration fields are given as follows:

$$
\boldsymbol{v}^{\alpha}\left(\boldsymbol{X}^{\alpha}, t\right)=\frac{\partial}{\partial t} \boldsymbol{\varphi}^{\alpha}\left(\boldsymbol{X}^{\alpha}, t\right) \text { and } \boldsymbol{a}^{\alpha}\left(\boldsymbol{X}^{\alpha}, t\right)=\frac{\partial}{\partial t} \boldsymbol{v}^{\alpha}\left(\boldsymbol{X}^{\alpha}, t\right) .
$$

A fundamental quantity in finite strain kinematics is the deformation gradient $\boldsymbol{F}^{\alpha}$ which is defined as the material gradient of the nonlinear deformation map:

$$
\boldsymbol{F}^{\alpha}=\nabla_{\boldsymbol{X}^{\alpha}} \boldsymbol{\varphi}^{\alpha}\left(\boldsymbol{X}^{\alpha}, t\right) .
$$

Hence, the mechanical compatibility condition $\operatorname{Curl}_{\alpha} \boldsymbol{F}^{\alpha}=\mathbf{0}$ is a priori fulfilled. In order to ensure a bijective relation between referential and current position and to avoid selfpenetration of the body, the Jacobian $J^{\alpha}=\operatorname{det} \boldsymbol{F}^{\alpha}>0$ of the deformation gradient has to be strictly positive. In order to describe deformations without being influenced by rigid body motions, the right Cauchy-Green tensor $\boldsymbol{C}^{\alpha}$ and left Cauchy-Green tensor $\boldsymbol{b}^{\alpha}$ can be introduced as follows:

$$
\boldsymbol{C}^{\alpha}=\boldsymbol{F}^{\alpha T} \cdot \boldsymbol{F}^{\alpha} \text { and } \boldsymbol{b}^{\alpha}=\boldsymbol{F}^{\alpha} \cdot \boldsymbol{F}^{\alpha T} .
$$

For the sake of simplicity and a more compact notation, we will drop the superscript $s$ for quantities referring to the solid component, whereas quantities with the superscript $\beta$ refer to component $\beta=\{f, m\}$.

\subsubsection{Field equations}

In this section, we derive all required field equations to describe fluid and particle transport in a magnetoactive solid skeleton material. Within the developed macroscopic theory, the solid skeleton is chosen as a reference component. The field equations are formulated in the Lagrangian description of the solid skeleton.

\subsubsection{Maxwell equations}

The reduced form of Maxwell's equations for the magnetostatic case in absence of free electric currents is given by Gauss's law for magnetism and Ampère's law; 


$$
\operatorname{Div} \mathbb{B}=0 \text { and } \operatorname{Curl} \mathbb{H}=\mathbf{0} \text {, }
$$

where $\mathbb{B}$ denotes the Lagrangian magnetic induction and $\mathbb{H}$ the Lagrangian magnetic field strength ${ }^{1}$. At a surface of material discontinuity $\mathcal{S}_{d}$, the corresponding jump conditions read as follows:

$$
\llbracket \mathbb{B} \rrbracket \cdot \boldsymbol{N}=0 \text { and } \llbracket \mathbb{H} \rrbracket \times \boldsymbol{N}=\mathbf{0} .
$$

Here, $\llbracket(\cdot) \rrbracket=(\cdot)^{+}-(\cdot)^{-}$denotes the jump of a quantity with regard to direction of the normal vector $\boldsymbol{N}$ to the interface $\mathcal{S}_{d}$. In a material body, the magnetic quantities are linked via the following general relation:

$$
\mathbb{B}=\mu_{0} J C^{-1} \cdot(\mathbb{H}+\mathbb{M}),
$$

where $\mathbb{M}$ denotes the Lagrangian magnetization and $\mu_{0}$ the magnetic permeability of free space. By the introduction of a magnetic scalar potential $\phi$ such that

$$
\mathbb{H}=\nabla_{X} \phi,
$$

Ampère's law is a priori satisfied. The continuity condition across a material interface associated with the potential reads

$$
\llbracket \phi \rrbracket=0 .
$$

\subsubsection{Balance of mass}

The conservation of mass is formulated separately for each component of the multiphase mixture. For the solid phase as a closed thermodynamical system, the local Lagrangian form of the balance of mass is given as follows:

$$
\rho_{0}^{s}=J \rho^{s}
$$

\section{Geometric transformations between Lagrangian and Eulerian tensor fields}

The geometrical mappings of infinitesimal line, area and volume elements between the reference and the current configuration are given as follows:

$$
\mathrm{d} \boldsymbol{x}=\mathbf{F} \cdot \mathrm{d} \boldsymbol{X}, \quad \mathrm{d} \boldsymbol{a}=\operatorname{cof} \mathbf{F} \cdot \mathrm{d} \boldsymbol{A} \text { and } \mathrm{d} v=J d V .
$$

The push-forward operations for the magnetic field quantities read as follows:

$$
\mathbb{b}=\mathbb{B} \cdot \operatorname{cof} \boldsymbol{F}^{-1}, \quad \mathbb{b}=\mathbb{H} \cdot \boldsymbol{F}^{-1} \text { and } \quad \mathbb{a n}=\mathbb{M} \cdot \boldsymbol{F}^{-1} \text {. }
$$

A Piola transformation of the first total Piola-Kirchhoff stress tensor leads to the symmetrical total Cauchy stress tensor

$$
\boldsymbol{\sigma}^{\text {tot }}=\boldsymbol{P}^{\text {tot }} \cdot \operatorname{cof} \boldsymbol{F}^{-1}
$$


where $\rho_{0}^{s}$ and $\rho^{s}$ denote the initial and current partial mass density of the solid, respectively. The partial mass density is linked to effective mass density $\rho_{e}^{s}$ via $\rho^{s}=\rho_{e}^{s} n^{s}$, where $n^{s}=\frac{\mathrm{d} v^{s}}{\mathrm{~d} v}$ is the volume fraction of the solid component. Next, consider the conservation of mass of component $\beta$. By choosing a Lagrangian description of the solid skeleton, the considered representative volume element is thermodynamically open with respect to component $\beta$. Therefore, the local form of the conservation of mass of component $\beta$ reads as follows:

$$
\dot{m}^{\beta}=-\operatorname{Div} \boldsymbol{J}^{\beta},
$$

in terms of the partial mass density of component $\beta$ per unit reference volume of the solid, which is related to the current partial mass density $\rho^{\beta}$ via $m^{\beta}=J \rho^{\beta}$. The operator $\dot{(\cdot)}=\left.\frac{\partial}{\partial t}(\cdot)\right|_{\boldsymbol{X}}$ defines the material time derivate with respect to the solid phase. Furthermore, $\boldsymbol{J}^{\beta}=m^{\beta} \boldsymbol{F}^{-1} \cdot\left(\boldsymbol{v}^{\beta}-\boldsymbol{v}\right)$ denotes the material mass flux vector of component $\beta$.

\subsubsection{Balance of linear and angular momentum}

The local form of the balance of linear and angular momentum for the multicomponent body reads as follows:

$$
\rho_{0}^{s} \boldsymbol{a}+\sum_{\beta} m^{\beta} \boldsymbol{a}^{\beta}=\operatorname{Div} \boldsymbol{P}^{\mathrm{tot}}+\left(\rho_{0}^{s}+\sum_{\beta} m^{\beta}\right) \boldsymbol{g} \text { and } \operatorname{skew}\left[\boldsymbol{P}^{\mathrm{tot}} \cdot \boldsymbol{F}^{T}\right]=\mathbf{0},
$$

where $\boldsymbol{g}$ is the gravitational acceleration and $\boldsymbol{P}^{\text {tot }}$ is the total first Piola-Kirchhoff stress tensor, which contains the contributions of the additional ponderomotive body force and body couple arising in magnetomechanics. The total first Piola-Kirchhoff stress tensor can be additively decomposed

$$
\boldsymbol{P}^{\text {tot }}=\boldsymbol{P}+\boldsymbol{P}^{\text {pon }}
$$

into a mechanical contribution $\boldsymbol{P}$ and a pondermotive contribution $\boldsymbol{P}^{\text {pon }}$, respectively. The specific form of the ponderomotive stress tensor is nonunique and depends on the chosen theory of field-matter interaction, see the studies by Pao [32] and Hutter et al. [33]. For the statistical model by de Groot and Suttorp [29] in the nonrelativistic approximation, the ponderomotive first Piola-Kirchhoff stress tensor takes the following form:

$$
\boldsymbol{P}^{\text {pon }}=\frac{1}{\mu_{0} J}\left[\boldsymbol{F} \cdot \mathbb{B} \otimes \mathbb{B}-\frac{1}{2}[\boldsymbol{C}:(\mathbb{B} \otimes \mathbb{B})] \boldsymbol{F}^{-T}\right]+(\mathbb{M} \cdot \mathbb{B}) \boldsymbol{F}^{-T}-\boldsymbol{F}^{-T} \cdot \mathbb{M} \otimes \mathbb{B} .
$$

Furthermore, the ponderomotive first Piola-Kirchhoff stress tensor $\boldsymbol{P}^{\text {pon }}=\boldsymbol{P}^{\text {mag }}+\boldsymbol{P}^{\text {max }}$ can be separated into a magnetization stress

$$
\boldsymbol{P}^{\mathrm{mag}}=(\mathbb{M} \cdot \mathbb{B}) \boldsymbol{F}^{-T}-\boldsymbol{F}^{-T} \cdot \mathbb{M} \otimes \mathbb{B}
$$

and a Maxwell stress tensor 


$$
\boldsymbol{P}^{\max }=\frac{1}{\mu_{0} J}\left[\boldsymbol{F} \cdot \mathbb{B} \otimes \mathbb{B}-\frac{1}{2}[\boldsymbol{C}:(\mathbb{B} \otimes \mathbb{B})] \boldsymbol{F}^{-T}\right]
$$

resulting in a symmetrical Cauchy-like Maxwell stress tensor when pushed forward to the current configuration via the Piola transformation (25.8). Note that the Maxwell stress also exists in free space, whereas all other stress contributions vanish in vacuum. The jump conditions at a surface of material discontinuity are given as follows:

$$
\llbracket \boldsymbol{P}^{\text {tot }} \rrbracket \cdot \boldsymbol{N}=-\boldsymbol{T},
$$

where $\boldsymbol{T}$ denotes the nominal mechanical traction vector.

\subsubsection{Dissipation inequality}

The dissipation inequality postulates that the temporal change in the free energy of the multicomponent system must be less than the power of the external actions. The local form of the Clausius-Duhem inequality under isothermal conditions reads

$$
\mathcal{D}=-\dot{\Psi}+\boldsymbol{P}^{\mathrm{tot}}: \dot{\boldsymbol{F}}+\mathbb{H} \cdot \dot{\mathbb{B}}+\sum_{\beta} \mu^{\beta} \dot{m}^{\beta}+\sum_{\beta}\left[-\nabla_{\boldsymbol{X}} \mu^{\beta}+\boldsymbol{F}^{T} \cdot\left(\mathbf{g}-\boldsymbol{a}^{\beta}\right)\right] \cdot \boldsymbol{J}^{\beta} \geq 0,
$$

in terms of a total energy density function $\Psi$ per unit reference volume. Here, $\mu^{\beta}$ denotes the chemical potential of component $\beta$, which is the conjugate variable to the partial mass density $\mathrm{m}^{\beta}$ and driving force for the evolution of $\mathrm{m}^{\beta}$. For a more detailed derivation of (25.21), we refer to Gebhart and Wallmersperger [42]. The dissipation inequality can be split into a local and a convective part as follows:

$$
\begin{gathered}
\mathcal{D}_{\text {loc }}=-\dot{\Psi}+\boldsymbol{P}^{\text {tot }}: \dot{\boldsymbol{F}}+\mathbb{H} \cdot \dot{\mathbb{B}}+\sum_{\beta} \mu^{\beta} \dot{m}^{\beta} \geq 0 \\
\mathcal{D}_{\text {con }}=\sum_{\beta}\left[-\nabla_{\boldsymbol{X}} \mu^{\beta}+\boldsymbol{F}^{T} \cdot\left(\mathbf{g}-\boldsymbol{a}^{\beta}\right)\right] \cdot \boldsymbol{J}^{\beta} \geq 0
\end{gathered}
$$

leading to sharper restrictions to the thermodynamical consistency of the constitutive equations.

\subsubsection{Constitutive modeling}

In the following, we will restrict ourselves to the constitutive modeling of porous magnetoactive gels with local nondissipative material behavior and without mobile particles. The constitutive relations that connect the total stress tensor, magnetic field strength and chemical potential of the fluid with its dual variables in the material configuration are provided by the evaluation of the local dissipation inequality according to Coleman and Noll [50]. We consider an enthalpic constitutive arrangement 
such that the total first Piola-Kirchhoff stress tensor $\boldsymbol{P}^{\text {tot }}$, the Lagrangian magnetic induction $\mathbb{B}$ and the chemical potential of the fluid $\mu^{f}$ are given as follows:

$$
\boldsymbol{P}^{\mathrm{tot}}=\frac{\partial \tilde{\Psi}\left(\boldsymbol{C}, \mathbb{H}, m^{f}\right)}{\partial \boldsymbol{F}}, \quad \mathbb{B}=-\frac{\partial \tilde{\Psi}\left(\boldsymbol{C}, \mathbb{H}, m^{f}\right)}{\partial \mathbb{H}} \quad \text { and } \quad \mu^{f}=\frac{\partial \tilde{\Psi}\left(\boldsymbol{C}, \mathbb{H}, m^{f}\right)}{\partial m^{f}}
$$

in terms of an objective total energy-enthalpy density function $\tilde{\Psi}\left(\boldsymbol{C}, \mathbb{H}, m^{f}\right)$ per unit reference volume. We restrict ourselves to an isotropic material response. For isotropic materials, the material symmetry condition is given as follows:

$$
\tilde{\Psi}\left(\boldsymbol{Q} \cdot \boldsymbol{C} \cdot \boldsymbol{Q}^{T}, \boldsymbol{Q} \cdot \mathbb{H}, m^{f}\right)=\tilde{\Psi}\left(\boldsymbol{C}, \mathbb{H}, m^{f}\right) \quad \forall \boldsymbol{Q} \in \mathcal{S O}(3) .
$$

Furthermore, we assume an additive decomposition of the total energy-enthalpy density function into a purely elastic part, a poroelastic part, a magnetoelastic part and a free space contribution as follows:

$$
\tilde{\Psi}\left(\boldsymbol{C}, \mathbb{H}, m^{f}\right)=\tilde{\Psi}_{\text {elas }}(\boldsymbol{C})+\tilde{\Psi}_{\text {poro }}\left(\boldsymbol{C}, m^{f}\right)+\tilde{\Psi}_{\text {mag }}(\boldsymbol{C}, \mathbb{H})+\tilde{\Psi}_{\text {vac }}(\boldsymbol{C}, \mathbb{H}) .
$$

\subsubsection{Elastic contribution}

The purely elastic behavior of the solid skeleton is characterized by a three parametric polyconvex neo-Hookean material model:

$$
\tilde{\Psi}_{\text {elas }}(\boldsymbol{C})=\frac{G}{2}\left[\left(\|\boldsymbol{F}\|^{2}-3\right)-2 \ln J\right]+\frac{\kappa}{\beta^{2}}\left(\beta \ln J+J^{-\beta}-1\right),
$$

where $G>0$ and $\kappa>0$ denote the shear and bulk modulus in the reference configuration, respectively, while $\beta>0$ is an empirical coefficient, see the study by Ogden and Hill [51].

\subsubsection{Poroelastic contribution}

The energy contribution of the pore fluid is given by a model adapted from the classical small-strain theory of Biot [45] as follows:

$$
\tilde{\Psi}_{\text {poro }}\left(\boldsymbol{C}, m^{f}\right)=\frac{M}{2}\left[b(J-1)-\frac{m^{f}}{\rho_{e}^{f}}\right]^{2}
$$

where $M$ and $b$ denote the Biot modulus and the Biot coefficient, respectively. Approximations for the Biot modulus and Biot coefficient are given in the works of Rice and Cleary [52] and Coussy [53]. More complex constitutive models to describe the poromechanical behavior can be found in e.g. the studies by Gajo and Denzer [54], Nedjar [55] and Voung et al. [56]. It should be mentioned that a Biot modulus $M \rightarrow \infty$ and a Biot coefficient $b=1$ correspond to an incompressible solid phase in case of an also incompressible fluid phase. 


\subsubsection{Magnetoelastic contribution}

For the magnetoelastic contribution, we choose an isotropic tensor function based on one purely magnetic and two coupling invariants, which reads as follows:

$$
\tilde{\Psi}_{\text {mag }}(\boldsymbol{C}, \mathbb{H})=-\frac{1}{2} \mu_{0}\left[\alpha_{1} \boldsymbol{C}^{-1}:(\mathbb{H} \otimes \mathbb{H})+\alpha_{2} \boldsymbol{C}^{-2}:(\mathbb{H} \otimes \mathbb{H})+\alpha_{3}|\mathbb{H}|^{2}\right] .
$$

Here, $\alpha_{1}, \alpha_{2}$ and $\alpha_{3}$ denote dimensionless material parameters. This phenomenological constitutive model is only able to accurately capture the material behavior at moderate magnetic fields. If saturation effects at higher magnetic fields should be captured sufficiently, more complex energy-enthalpy density functions should be used, see e.g. the constitutive models for magnetorheological elastomers presented in the studies by Lefèvre et al. [57] and Mukherjee et al. [58].

\subsubsection{Free space contribution}

The enthalpy stored in the underlying free space - where the multicomponent mixture is embedded in - is given as follows:

$$
\tilde{\Psi}_{\text {vac }}(\boldsymbol{C}, \mathbb{H})=-\frac{1}{2} \mu_{0} J \boldsymbol{C}^{-1}:(\mathbb{H} \otimes \mathbb{H}) .
$$

\subsubsection{Material fluid mass flux vector}

The material fluid mass flux vector is defined by the constitutive law

$$
\boldsymbol{J}^{f}=\partial_{\mathfrak{B}} \tilde{\Phi}(\boldsymbol{C}, \boldsymbol{B})
$$

in terms of an objective dissipation density function $\tilde{\Phi}$ that has to be convex and smooth in $\boldsymbol{B}=\left[-\nabla_{\boldsymbol{X}} \mu^{f}+\boldsymbol{F}^{T} \cdot\left(\mathbf{g}-\boldsymbol{a}^{f}\right)\right]$, which can be interpreted as the fluid driving force. Assuming Stokes flow on the microscale, we describe the fluid flow through the porous solid skeleton by a spatial isotropic Darcy-type flow governed by the following equation:

$$
\tilde{\Phi}(\boldsymbol{C}, \boldsymbol{B})=\frac{1}{2}\left[\rho_{e}^{f}\right]^{2} k J \boldsymbol{C}^{-1}:(\boldsymbol{B} \otimes \mathfrak{B}),
$$

where $k>0$ denotes the spatial hydraulic permeability.

\subsubsection{Variational formulation}

The solution of the initial boundary value problem in the quasi-static case, where mechanical inertia effects and time-dependent electromagnetic couplings are neglected, is governed by a rate-type variational principle whose time-discrete formulation is given as follows: 


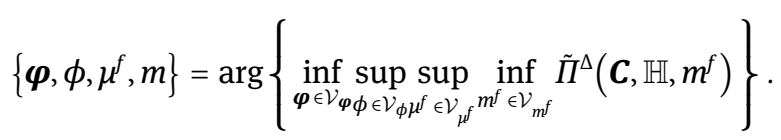

It determines the deformation map of the solid skeleton $\boldsymbol{\varphi}$, the magnetic scalar potential $\phi$, the partial mass density of the fluid $m^{f}$ and the chemical potential of the fluid $\mu^{f}$ at current time $t$ as a saddlepoint of the time-discrete potential $\tilde{\Pi}^{\Delta}$. In the absence of external loading contributions, the time-discrete potential $\tilde{\Pi}^{\Delta}$ is given as follows:

$$
\tilde{\Pi}^{\Delta}\left(\boldsymbol{C}, \mathbb{H}, \mu^{f}, m^{f}\right)=\int_{\mathcal{B}} \tilde{\Psi}\left(\boldsymbol{C}, \mathbb{H}, m^{f}\right)-\Delta t \tilde{\Phi}\left(\boldsymbol{C}_{n}, \boldsymbol{B}\right)-\mu^{f}\left(m^{f}-m_{n}^{f}\right) d V,
$$

within the time interval $\left[t_{n}, t\right]$ with length $\Delta t=t-t_{n}$. Note that the dissipation density function is evaluated at a frozen deformation at time $t_{n}$. The admissable trial solution spaces for the primary variables are given as follows:

$$
\begin{aligned}
& \mathcal{V}_{\boldsymbol{\varphi}}:=\left\{\boldsymbol{\varphi} \in H^{1}(\mathcal{B}) \mid \boldsymbol{\varphi}=\overline{\boldsymbol{\varphi}} \quad \text { on } \partial \mathcal{B}_{\boldsymbol{\varphi}}\right\}, \\
& \mathcal{V}_{\phi}:=\left\{\phi \in H^{1}(\mathcal{B}) \mid \phi=\bar{\phi} \quad \text { on } \partial \mathcal{B}_{\phi}\right\} \text {, } \\
& \mathcal{V}_{\mu^{f}}:=\left\{\mu^{f} \in H^{1}(\mathcal{B}) \mid \mu^{f}=\bar{\mu}^{f} \quad \text { on } \quad \partial \mathcal{B}_{\mu^{f}}\right\} \text {. } \\
& \mathcal{V}_{m^{f}}:=\left\{m^{f} \in L^{2}\right\}
\end{aligned}
$$

The saddlepoint problem (32) can be solved in a sequential manner. First, we introduce a reduced time-discrete potential

$$
\tilde{I}_{\text {red }}\left(\boldsymbol{C}, \mathbb{H}, \mu^{f}\right)=\int_{\mathcal{B}} \tilde{\pi}_{\text {red }}\left(\boldsymbol{C}, \mathbb{H}, \mu^{f}\right) d V
$$

through the local condensation of the partial mass density of the fluid $m^{f}$ given as follows:

$$
\tilde{\pi}_{\mathrm{red}}^{\mathrm{A}}\left(\boldsymbol{C}, \mathbb{H}, \mu^{f}\right)=\inf _{m^{f} \in \mathcal{V}_{m f}}\left[\tilde{\Psi}\left(\boldsymbol{C}, \mathbb{H}, m^{f}\right)-\Delta t \tilde{\Phi}\left(\boldsymbol{C}_{n}, \boldsymbol{B}\right)-\mu^{f}\left(m^{f}-m_{n}^{f}\right)\right],
$$

see also the studies by Böger et al. [59] and Teichtmeister et al. [60]. With the definition of the reduced time-discrete potential (25.35) at hand, the global solution of the initial boundary value problem is then given as follows:

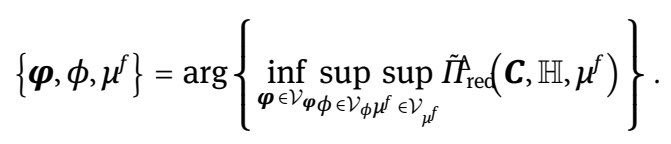

\subsubsection{Representative boundary value problems}

In this section, the presented macroscopic modeling framework is applied to investigate the magnetomechanical response of soft isotropic ferrogels at steady state. This numerical study is motivated by the experiments conducted in the studies by Diguet 
a)

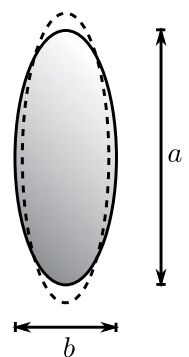

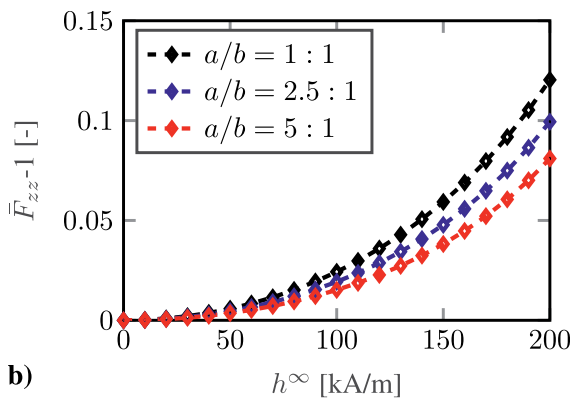

Figure 25.1: Macroscale modeling of ferrogels. (a) Boundary value problem and (b) macroscopic deformation response of initially spheroidal ferrogel specimens with aspect ratios of $a / b=\{1: 1$, $2.5: 1,5: 1\}$ in direction of the applied magnetic field.

et al. [61] and Bodelot et al. [62]. In detail, we consider spheroidal shaped specimens with aspect ratios of $a / b=\{1: 1,2.5: 1,5: 1\}$. This specific shape is chosen because only for ellipsoidal shaped bodies a homogeneous distribution of the magnetic fields within the specimen can be realized. The free space surrounding the material body is modeled as a hyperelastic pseudosolid with negligible elastic properties and magnetic properties identical to free space. Throughout the domain, a homogeneous external magnetic field is applied which is aligned in parallel with the major axis of the spheroid, see Figure 25.1a. The free space box surrounding the body is of sufficient size such that the magnetic self-field of the body vanishes on the boundary of the free space. Furthermore, we prescribe homogeneous Dirichlet boundary conditions for the chemical potential of the fluid on the surface of the ferrogel domain. The material parameters used for the numerical study are listed in Table 25.1.

In Figure 25.1b, the macroscopic deformation of the specimens in direction of the applied magnetic field is plotted over the external applied magnetic field strength.

Table 25.1: Macroscale modeling of ferrogels. Material parameters of the ferrogel.

\begin{tabular}{llrl}
\hline Parameter & Symbol & Value & Unit \\
\hline Shear modulus & $G$ & 25 & {$[\mathrm{kPa}]$} \\
Bulk modulus & $\mathrm{K}$ & 50 & {$[\mathrm{kPa}]$} \\
Elastic coefficient & $\beta$ & 1 & {$[-]$} \\
Biot modulus & $M$ & 100 & {$[\mathrm{kPa}]$} \\
Biot coefficient & $b$ & 1 & {$[-]$} \\
Effective mass density of the fluid & $\rho_{e}^{f}$ & 1000 & {$\left[\mathrm{~kg} / \mathrm{m}^{3}\right]$} \\
Hydraulic permeability coefficient & $k$ & 900 & {$\left[\mathrm{~mm}^{2} / \mathrm{MPas}\right]$} \\
Permeability of free space & $\mu_{0}$ & $4 \pi \times 10^{-7}$ & {$\left[\mathrm{~N} / \mathrm{A}^{2}\right]$} \\
Magnetic material parameter & $\alpha_{1}$ & 0.35 & {$[-]$} \\
Magnetic material parameter & $\alpha_{2}$ & 0.1 & {$[-]$} \\
Magnetic material parameter & $\alpha_{3}$ & 0.15 & {$[-]$} \\
\hline
\end{tabular}


For all investigated specimen aspect ratios, a positive magnetostriction is observable which is quadratically dependent on the applied magnetic field. The magnetostrictive effect increases with decreasing aspect ratio and reaches its maximum for an aspect ratio of $a / b=1: 1$. This is in line with the analytical model based on the concept of demagnetizing energy proposed by Raikher and Stolbov [63, 64].

The spatial distribution of the deformation gradient component $F_{z z}$ in the rotational symmetry plane for all three specimens at an external magnetic field strength of $h^{\infty}=200 \mathrm{kA} / \mathrm{m}$ is depicted in Figure 25.2a. In the initially spherical shaped specimen, we observe the development of a boundary layer where the deformation is significantly lower than in the center of the specimen. For higher aspect ratios, the region of maximal deformation shifts to the tips of the specimens at both ends of the major axis, while the field distribution of the deformation gradient component $F_{z z}$ is nearly homogeneous along the minor axis of the bodies. These field distributions of $F_{z z}$ - caused by the jump of the magnetic field quantities at the boundary of the material body seem to be characteristic for this specific load case as these deformation patterns were also reported in the numerical studies of Keip and Rambausek [36] and Kalina et al. [65]. Due to the induced deformation, the shape of the specimen differs from an exact spheroid to some extend. This consequently results in an inhomogeneous magnetization field $m_{z}$ within the bodies, see Figure 25.2b. For all three specimens, the region where the magnetization component $m_{z}$ reaches its minimum is located at both ends of the major axis. Furthermore, it can be seen that aspect ratios far from $a / b=1: 1$ lead to significantly smaller deviations of the magnetization component $m_{z}$ in the center of the body and along its minor axis. The spatial distribution of the mechanical Cauchy stress tensor component $\sigma_{z z}$ in all three specimens is depicted in Figure 25.2c. It was shown by Kalina et al. [65] that the mechanical stress tensor is a crucial quantity when it comes to the fitting of macroscopic constitutive models for magnetoactive polymers. For all three specimens, the maximum of the mechanical cauchy stress $\sigma_{z z}$ is located at the ends of the major axis. Furthermore, we observe that larger aspect ratios lead to a significantly higher field homogeneity of $\sigma_{z z}$ in the center of the specimen and along its minor axis. This is due to the shape of the ponderomotive traction vector $\boldsymbol{t}^{\text {pon }}=\llbracket \boldsymbol{\sigma}^{\text {pon }} \rrbracket \cdot \boldsymbol{n}$ at the interface between the free space and the material body which strongly depends upon the geometry of the body. The mechanical stress tensor field intrinsically depends upon the pondermotive traction vector as $\boldsymbol{\sigma} \cdot \boldsymbol{n}=\boldsymbol{t}^{\text {pon }}$ holds on the boundary of the body in absence of mechanical tractions.

These results show that for spheroidal shaped specimens, aspect ratios far from $a$ / $b=1: 1$ lead to significantly more homogeneous field distributions within the body. Thus, prolate spheroids are preferable in an experimental setup. Furthermore, a relaxation of the assumption of field homogeneity in the postprocessing of experimental data would lead to a more accurate reconstruction of the internal magnetization and stress state of the material body, as proposed by Keip and Rambausek [36]. 


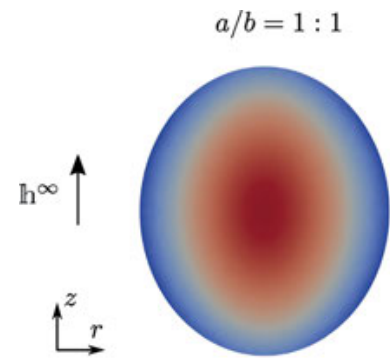

a)

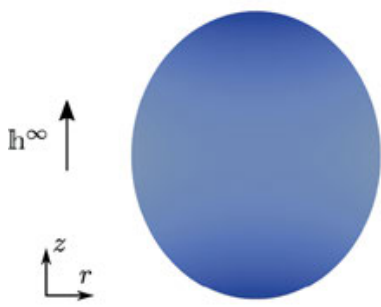

b)

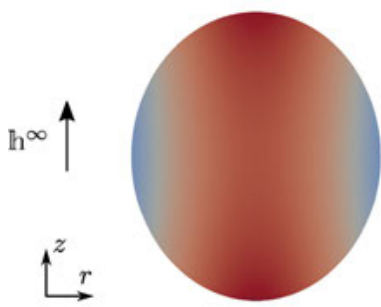

c) $a / b=2.5: 1$

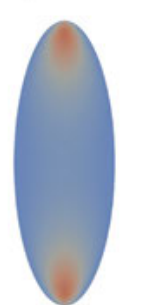

$$
F_{z z}[-]
$$
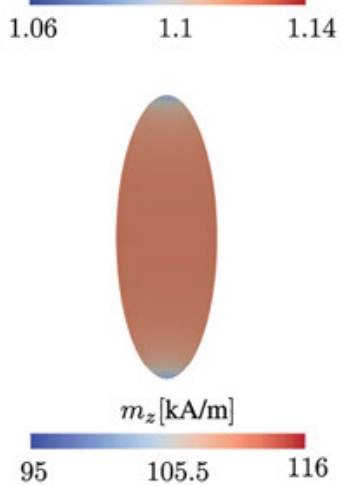

95

105.5
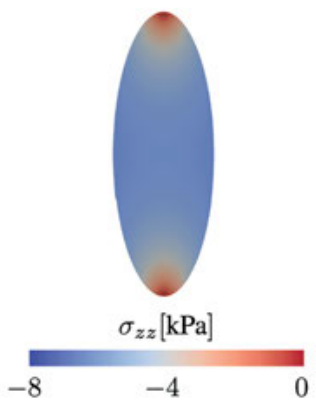

$a / b=5: 1$
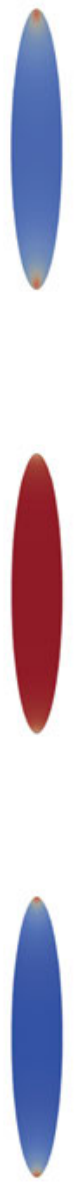

Figure 25.2: Macroscale modeling of ferrogels. Spatial distribution of (a) the deformation gradient component $F_{z z}$, (b) the magnetization component, $m_{z}(\mathrm{c})$ the mechanical cauchy stress component $\sigma_{z z}$ in the initially spheroidal ferrogel specimens with aspect ratios of $a / b=\{1: 1,2.5: 1,5: 1\}$ at an external magnetic field strength $\mathrm{ln}^{\infty}=h^{\infty} \boldsymbol{e}_{z}$ with $h^{\infty}=200 \mathrm{kA} / \mathrm{m}$.

\subsection{Microscale modeling of ferrogels}

In this section, we propose a microscopic continuum-based framework for the modeling of ferrogels with nondissipative material behavior. Assuming separation of length scales, this modeling approach is embedded into a suitable energy-based 
computational homogenization scheme following Chatzigeorgiou et al. [35] in order to bridge between microscale and macroscale. This modeling technique allows us to analyze the effective material behavior of the microstructure at a single material point of the macrostructure without the influence of any macrostructural effects.

\subsubsection{Kinematics}

Consider a representative volume element $\mathcal{R} \mathcal{V E}$ consisting of a fixed set of material particles $P \in \mathcal{R} \mathcal{V} \mathcal{E}$ embedded in the Euclidean space at time $t \in \mathbb{R}^{+}$. Let its reference configuration be denoted by $\mathcal{B} \subset \mathbb{R}^{3}$ and its current configuration by $\mathcal{S} \subset \mathbb{R}^{3}$. If $\boldsymbol{X} \in \mathcal{B}$ and $\boldsymbol{x} \in \mathcal{S}$ denote the referential and current position of a material point $P$, respectively, in the Euclidian space, the deformation $\boldsymbol{\varphi}$ is the smooth bijective mapping

$$
\boldsymbol{\varphi}:=\left\{\begin{array}{l}
\mathcal{B} \times T \rightarrow \mathcal{S} \subset \mathbb{R}^{3} \\
(\boldsymbol{X}, t) \mapsto \boldsymbol{X}=\boldsymbol{\varphi}(\boldsymbol{X}, t)
\end{array}\right.
$$

of the material body onto a time sequence of configurations in space. The tensor field

$$
\boldsymbol{F}=\nabla_{\boldsymbol{X}} \boldsymbol{\varphi}(\boldsymbol{X}, t) .
$$

denotes the deformation gradient, which linearly maps material tangent vectors to associated deformed spatial tangent vectors.

\subsubsection{Field equations}

In the following, we summarize the local field equations for the quasi-static case of magnetomechanics. In the subsequent formulation, free current densities as well as mechanical body forces are neglected. With respect to a Lagrangian description in the reference configuration, the boundary value problem is governed by the following set of equations:

1. Gauss's law for magnetism

$\operatorname{Div} \mathbb{B}=0$

2. Ampère's law

Curl $\mathbb{H}=\mathbf{0}$

3. Balance of mass

$\rho_{0}=J \rho$

4. Balance of linear momentum

$\operatorname{Div} \boldsymbol{P}^{\text {tot }}=\mathbf{0}$

5. Balance of angular momentum

skew $\left[\boldsymbol{P}^{\text {tot }} \cdot \boldsymbol{F}^{T}\right]=\mathbf{0}$

6. Dissipation inequality

$-\dot{\Psi}+\boldsymbol{P}^{\text {tot }}: \dot{\boldsymbol{F}}+\mathbb{H} \cdot \dot{\mathbb{B}} \geq 0$

By introducing a magnetic vector potential $\boldsymbol{A}$ such that

$$
\mathbb{B}=\operatorname{Curl} \boldsymbol{A},
$$


Ampère's law is a priori satisfied. This definition - in combination with the continuity condition $(9)_{1}$ on the normal component of the magnetic induction - leads to a tangential continuity of the magnetic vector potential

$$
\llbracket \boldsymbol{A} \rrbracket \times \boldsymbol{N}=\mathbf{0}
$$

across a material interface.

\subsubsection{Constitutive modeling}

We consider an energetic constitutive arrangement such that the microscopic total first Piola-Kirchhoff stress tensor $\boldsymbol{P}^{\text {tot }}$ and the Lagrangian magnetic field strength $\mathbb{H}$ are given as follows:

$$
\boldsymbol{P}^{\mathrm{tot}}=\frac{\partial \Psi(\boldsymbol{C}, \mathbb{B})}{\partial \boldsymbol{F}} \text { and } \mathbb{H}=\frac{\partial \Psi(\boldsymbol{C}, \mathbb{B})}{\partial \mathbb{B}}
$$

in terms of an objective total energy density function $\Psi(\boldsymbol{C}, \mathbb{B})$. Following the concept of Miehe et al. [66] and Ethiraj and Miehe [67], we assume that the total energy density function $\Psi$ is polyconvex with respect to the deformation gradient $\boldsymbol{F}$ for $\mathbb{B}=\mathbf{0}$ and convex with respect to the Lagrangian magnetic induction $\mathbb{B}$.

We restrict ourselves to an isotropic material response of the constituents. For isotropic materials, the material symmetry condition is given as follows:

$$
\Psi\left(\boldsymbol{Q} \cdot \boldsymbol{C} \cdot \boldsymbol{Q}^{T}, \boldsymbol{Q} \cdot \mathbb{B}\right)=\Psi(\boldsymbol{C}, \mathbb{B}) \quad \forall \boldsymbol{Q} \in \mathcal{S O}(3) .
$$

Furthermore, we assume an additive decomposition of the total energy density function into a purely elastic part, a magnetic particle part and a free space contribution as follows:

$$
\Psi(\boldsymbol{C}, \mathbb{B})=\Psi_{\text {elas }}(\boldsymbol{C})+\Psi_{\text {mag }}(\mathbb{B})+\Psi_{\text {vac }}(\boldsymbol{C}, \mathbb{B})
$$

\subsubsection{Elastic contribution}

The purely elastic behavior of the constituents is modeled by a two parametric neoHookean material model as follows:

$$
\Psi_{\text {elas }}(\boldsymbol{C})=\frac{1}{2}\left[G\left(\|\boldsymbol{F}\|^{2}-\ln J^{2}-3\right)+\frac{\lambda}{2}\left(J^{2}-\ln J^{2}-1\right)\right],
$$

where $\lambda>0$ and $G>0$ denote the first and second Lamé parameter, respectively, see the study by Ciarlet [68]. In the small strain regime, these are linked to the Young's modulus $E$ and Poisson's ratio $v$ via $\lambda=\frac{v E}{(1+v)(1-2 v)}$ and $G=\frac{E}{2(1+v)}$. 


\subsubsection{Magnetic contribution}

The soft magnetic behavior of the ferromagnetic particles is described by a Langevintype model, which reads as follows:

$$
\Psi_{\text {mag }}(\mathbb{B})=\frac{\mu_{0} m_{s}^{2}}{3 \chi}\left\{\ln \left[\frac{3 \chi}{\mu_{0} m_{s}}|\mathbb{B}|\right]-\ln \left[\sinh \left(\frac{3 \chi}{\mu_{0} m_{s}}|\mathbb{B}|\right)\right]\right\} .
$$

Here, $m_{s}$ denotes the magnetic saturation and $\chi$ the magnetic susceptibility which is linked to the relative magnetic permeability via $\mu_{r}=1 /(1-\chi)$. Note that there are more than one definitions of magnetic susceptibility in the literature.

\subsubsection{Free space contribution}

The energy stored in the underlying free space - where the $\mathcal{R} \mathcal{V E}$ is embedded in - is given as follows:

$$
\Psi_{\text {vac }}(\boldsymbol{C}, \mathbb{B})=\sup _{\mathbb{H}}\left[\tilde{\Psi}_{\text {vac }}(\boldsymbol{C}, \mathbb{H})+\mathbb{B} \cdot \mathbb{H}\right]=\frac{1}{2} \mu_{0}^{-1} J^{-1} \boldsymbol{C}:(\mathbb{B} \otimes \mathbb{B}) .
$$

\subsubsection{Homogenization framework}

Computational homogenization schemes enable to bridge scales between microscale and macroscale by the application of suitable micro-to-macro transition concepts, linking the microscopic response of the heterogeneous microstructure directly with the macroscopic overall response in a homogenized sense. Within this work, we make use of an energy-based formulation based on the unified magnetomechanical homogenization framework outlined in the study by Chatzigeorgiou et al. [35].

\subsubsection{Definition of macrovariables}

The macroscopic Lagrangian magnetic induction $\overline{\mathbb{B}}$ and magnetic field strength $\overline{\mathbb{H}}$ are related to their microscopic counterparts via averages over the volume or the boundary of the $\mathcal{R} \mathcal{V E}$ as follows:

$$
\begin{aligned}
\overline{\mathbb{B}} & =\frac{1}{V_{0}} \int_{\mathcal{B}} \mathbb{B} d V=\frac{1}{V_{0}} \int_{\partial \mathcal{B}} \boldsymbol{N} \times \boldsymbol{A} d A \text { and } \\
\overline{\mathbb{H}} & =\frac{1}{V_{0}} \int_{\mathcal{B}} \mathbb{H} d V=\frac{1}{2} \frac{1}{V_{0}} \int_{\partial \mathcal{B}} \boldsymbol{X} \times \mathbb{H} \times \boldsymbol{N} d A .
\end{aligned}
$$

In analogy, the macroscopic deformation gradient $\overline{\boldsymbol{F}}$ and the total first Piola-Kirchhoff stress tensor $\overline{\boldsymbol{P}}^{\text {tot }}$ are defined as follows: 


$$
\begin{aligned}
\overline{\boldsymbol{F}} & =\frac{1}{V_{0}} \int_{\mathcal{B}} \boldsymbol{F} d V=\frac{1}{V_{0}} \int_{\partial \mathcal{B}} \boldsymbol{\varphi} \otimes \boldsymbol{N} d A \text { and } \\
\overline{\boldsymbol{P}}^{\mathrm{tot}} & =\frac{1}{V_{0}} \int_{\mathcal{B}} \boldsymbol{P}^{\mathrm{tot}} d V=\frac{1}{V_{0}} \int_{\partial \mathcal{B}} \boldsymbol{P}^{\mathrm{tot}} \cdot \boldsymbol{N} \otimes \boldsymbol{X} d A .
\end{aligned}
$$

\subsubsection{Hill-Mandel macrohomogeneity condition}

Following conceptually the study by Hill [69], the macrohomogenity condition (HillMandel lemma) in the magnetomechanical context is given as follows:

$$
\frac{1}{V_{0}} \int_{\mathcal{B}} \boldsymbol{P}^{\text {tot }}: \dot{\boldsymbol{F}}+\mathbb{H} \cdot \dot{\mathbb{B}} d V=\overline{\boldsymbol{P}}^{\text {tot }}: \dot{\overline{\boldsymbol{F}}}+\overline{\mathbb{H}} \cdot \dot{\overline{\mathbb{B}}}
$$

Note that we assume an additive decomposition of the primary microscopic variables into linear macroscopic contributions and superimposed fine-scale fluctuation fields

$$
\boldsymbol{\varphi}=\overline{\boldsymbol{F}} \cdot \boldsymbol{X}+\tilde{\boldsymbol{\varphi}} \text { and } \boldsymbol{A}=\frac{1}{2} \overline{\mathbb{B}} \times \boldsymbol{X}+\tilde{\boldsymbol{A}}
$$

The micro-macro consistency of power densities is satisfied by a suitable set of boundary conditions, i.e. periodic ones for the deformation map and the vector potential and antiperiodic ones for the total traction vector and the tangential magnetic field strength

$$
\begin{gathered}
\llbracket \boldsymbol{\varphi} \rrbracket=\overline{\boldsymbol{F}} \cdot \llbracket \boldsymbol{X} \rrbracket \text { and } \boldsymbol{T}^{\mathrm{tot}}\left(\boldsymbol{X}^{-}\right)=-\boldsymbol{T}^{\mathrm{tot}}\left(\boldsymbol{X}^{+}\right) \\
\llbracket \boldsymbol{A} \rrbracket=\frac{1}{2} \overline{\mathbb{B}} \times \llbracket \boldsymbol{X} \rrbracket \text { and } \mathbb{H} \times \boldsymbol{N}\left(\boldsymbol{X}^{-}\right)=-\mathbb{H} \times \boldsymbol{N}\left(\boldsymbol{X}^{+}\right)
\end{gathered}
$$

at corresponding points on opposing boundaries of the microstructure. Here, $\llbracket(\cdot) \rrbracket=$ $(\cdot)^{+}-(\cdot)^{-}$denotes the jump of a quantity with respect to opposite boundaries of the $\mathcal{R} \mathcal{V} \mathcal{E}$.

\subsubsection{Variational formulation}

The numerical implementation of the magnetomechanical boundary value problem is based on an energy formulation with periodic Dirichlet boundary conditions conceptually in line with the studies by Kalina et al. [19] and Miehe et al. [70]. The deformation map and the Lagrangian magnetic vector potential are determined by the variational principle as follows:

$$
\{\boldsymbol{\varphi}, \boldsymbol{A}\}=\arg \left\{\inf _{\boldsymbol{\varphi} \in \mathcal{V}_{\boldsymbol{\varphi}} \boldsymbol{A} \in \mathcal{V}_{\boldsymbol{A}}} \int_{\mathcal{B}} \Psi(\boldsymbol{C}, \mathbb{B}) d V\right\}
$$


In this minimization principle, the admissible function space for the deformation field and the magnetic vector potential satisfy the Dirichlet boundary conditions

$$
\begin{array}{ll}
\mathcal{V}_{\boldsymbol{\varphi}}:=\left\{\boldsymbol{\varphi} \in H^{1}(\mathcal{B}) \mid \llbracket \boldsymbol{\varphi} \rrbracket=\overline{\boldsymbol{F}} \cdot \llbracket \boldsymbol{X} \rrbracket\right. & \text { on } \quad \partial \mathcal{B}\} \\
\mathcal{V}_{\boldsymbol{A}}:=\left\{\boldsymbol{A} \in H(\operatorname{Curl}, \mathcal{B}) \mid \llbracket \boldsymbol{A} \rrbracket=\frac{1}{2} \overline{\mathbb{B}} \times \llbracket \boldsymbol{X} \rrbracket\right. & \text { on } \quad \partial \mathcal{B}\}
\end{array}
$$

on the surface $\partial B$ of the microstructure. In three dimensions, the solution of the boundary value problem (25.57) is nonunique in terms of the gauge symmetry transformation $\boldsymbol{A} \rightarrow \boldsymbol{A}+\nabla \varphi$. From a numerical point of view, this will lead to an illconditioned stiffness matrix. For a well-posed mathematical formulation, additional gauge conditions can be imposed on the vector potential, such as the Coulomb gauge enforcing $\operatorname{Div} \boldsymbol{A}=0$, see the study by Semenov et al. [71]. For a more comprehensive treatment of variational principles in magnetomechanics, we refer to the works of Vogel et al. [72, 73], Miehe et al. [70, 74], Bustamante et al. [75] and Šilhavý [76].

\subsubsection{Representative boundary value problems}

In this section, the microscale modeling framework is applied to investigate the effective material behavior of ferrogels with a random monodisperse particle distribution. In this study, we consider microporous ferrogels, where the characteristic length of the pores within the polymer matrix is much smaller than the size of the magnetizable particles. In detail, we analyze the effective material response of twodimensional unit cells with three different particle volume fractions $\phi=$ $\{10 \%, 20 \%, 30 \%\}$ for two representative combined magnetomechanical load states. The unit cells comprise approximately 500 magnetizable particles in order to obtain a representative volume element with an isotropic material response, see the studies on representativity of unit cells of Danas [21]. This determines the geometrical dimensions of the different unit cells. The chosen set of material parameters for the constituents used in this study is given in Table 25.2.

Table 25.2: Microscale modeling of ferrogels. Material parameters of the constituents.

\begin{tabular}{llrrl}
\hline Parameter & Symbol & Particle & Matrix & Unit \\
\hline Young's modulus & $E$ & $200 \times 10^{6}$ & 100 & {$[\mathrm{kPa}]$} \\
Poisson's ratio & $\mathrm{V}$ & 0.3 & 0.3 & {$[-]$} \\
Permeability of free space & $\mu_{0}$ & $4 \pi \times 10^{-7}$ & $4 \pi \times 10^{-7}$ & {$\left[\mathrm{~N} / \mathrm{A}^{2}\right]$} \\
Magnetic susceptibility & $X$ & 0.9 & 0 & {$[-]$} \\
Magnetic saturation & $m_{s}$ & 875 & - & {$[\mathrm{kA} / \mathrm{m}]$} \\
\hline
\end{tabular}




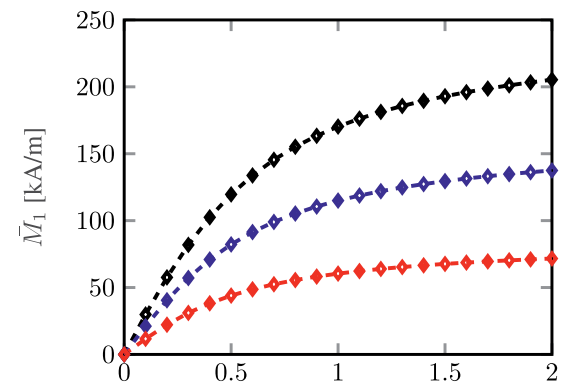

a)

$\bar{B}[\mathrm{~T}]$

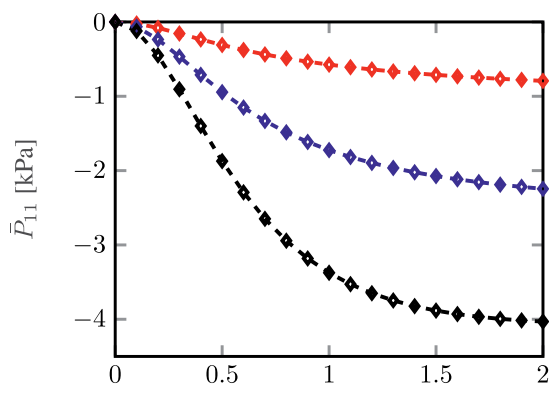

b)

$\bar{B}[\mathrm{~T}]$

- $-\phi=10 \%-\downarrow-\phi=20 \%-\downarrow-\phi=30 \%$

Figure 25.3: Microscale modeling of ferrogels. Effective material behavior of random monodisperse microstructures for load case I: (a) Magnetization $\bar{M}_{1}$ versus magnetic induction $\bar{B}$ and (b) mechanical stress $\bar{P}_{11}$ versus magnetic induction $\bar{B}$.

\subsubsection{Load Case I}

As a first load case, we consider a magnetically driven load state with a prevented macroscopic deformation of the microstructures characterized by

$$
[\bar{F}]=\left[\begin{array}{ll}
1 & 0 \\
0 & 1
\end{array}\right] \text { and }[\overline{\mathbb{B}}]=\left[\begin{array}{l}
\bar{B} \\
0
\end{array}\right]
$$

where the macroscopic magnetic induction $\bar{B}$ is linearly increased from $0 \mathrm{~T}$ to a maximum of $2 \mathrm{~T}$ within 20 increments. The effective magnetization behavior of the microstructures is depicted in Figure 25.3a. Due to the material isotropy of the unit cells - caused by the unstructured particle distribution within the polymer matrix - the magnetization $\bar{M}_{2}$ vanishes. Furthermore, a monotonic increase of the magnetization with increasing particle volume fraction can be observed. The magnetization responses of the different microstructures also indicate that the saturation magnetization of the composite is linearly dependent on the particle volume fraction and can be calculated via a simple rule of mixtures $\bar{m}_{s}=\phi m_{s}$. In Figure 25.3b, the macroscopic mechanical stress $\bar{P}_{11}$ is plotted over the applied magnetic induction. Initially, a quadratic dependency of the mechanical stress on the magnetic induction is observable, while at higher magnetic fields, saturation of the magnetization leads to a saturation of the mechanical stress as well.

Negative values of the mechanical stress $\bar{P}_{11}$ along with a prevented macroscopic deformation indicate the tendency of the unit cells to expand in the direction of the applied magnetic induction. This is in agreement with experimental observations in the study by Gollwitzer et al. [77] and Filipcsei and Zrínyi [78]. The spatial distribution of the local magnetic induction $b_{1}$ within the unit cells in Figure 25.4 exhibits considerably 
$\phi=10 \%$

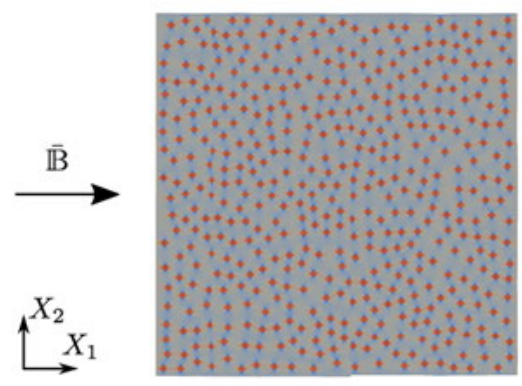

$\phi=20 \%$
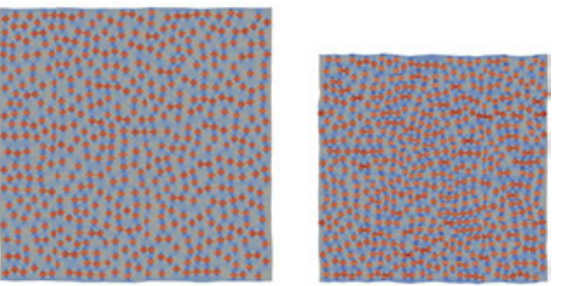

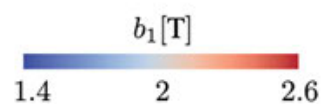

Figure 25.4: Microscale modeling of ferrogels. Spatial distribution of the local magnetic induction $b_{1}$ in the unit cells with particle volume fraction $\phi=\{10 \%, 20 \%, 30 \%\}$ at a macroscopic magnetic induction of $\bar{B}=\bar{B} e_{1}$ with $\bar{B}=2 \mathrm{~T}$.

stronger mutual particle interactions at higher particle volume fractions. This effect arises due to the decreasing particle distances with increasing particle volume fractions.

\subsubsection{Load Case II}

In the second load case, the microstructures are subjected to a combined magnetomechanical load state characterized by

$$
[\bar{F}]=\left[\begin{array}{cc}
1 & 0.1 \\
0 & 1
\end{array}\right] \text { and }[\overline{\mathbb{B}}]=\left[\begin{array}{l}
0 \\
\bar{B}
\end{array}\right]
$$

where - in analogy to the previous load case - the macroscopic magnetic induction $\bar{B}$ is linearly increased from $0 \mathrm{~T}$ to a maximum of $2 \mathrm{~T}$ within 20 increments. The macroscopic magnetization behavior of the microstructures is shown in Figure 25.5a. A comparison with the results of the previous load case given in Figure 25.3a indicates that the magnetization response is nearly independent of the macroscopic deformation state, see also the studies of Kalina et al. [19]. Due to the macroscopic simple shear deformation of the microstructure, the mechanical shear stress $\bar{P}_{12}$ is positive for all three unit cells at vanishing magnetic fields, see Figure 25.6b.

We observe an initial quadratic dependency of the mechanical shear stress $\bar{P}_{12}$ on the magnetic induction with saturation effects occurring at higher magnetic fields. The monotonic increase of the mechanical shear stress $\bar{P}_{12}$ with the magnetic induction for the unit cells with particle volume fractions of $\phi=\{10 \%, 20 \%\}$ at a fixed macroscopic simple shear deformation indicates a positive magnetorheological effect. This observation coincides with the experimental results in the study by Jolly et al. [79]. In 

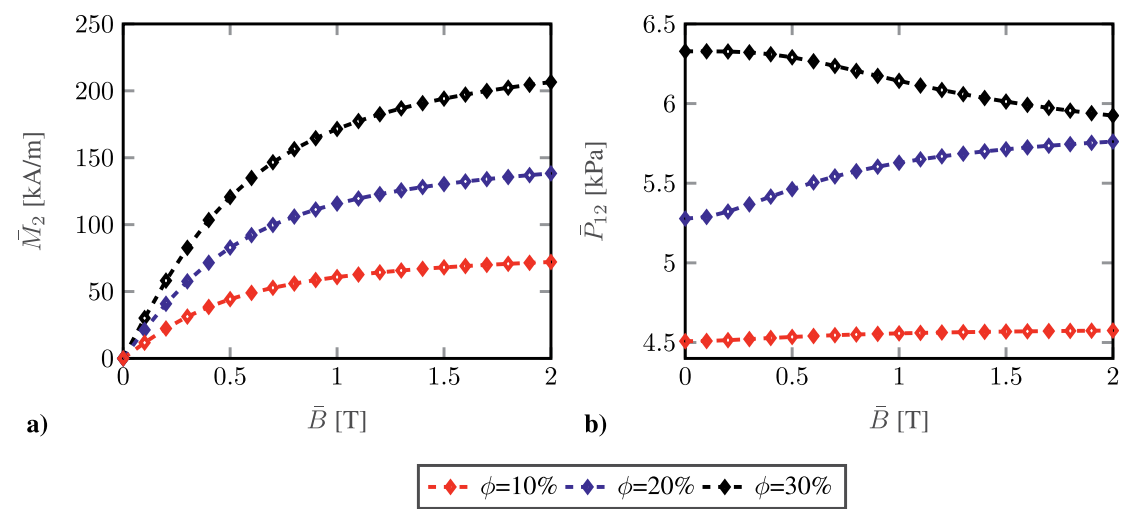

Figure 25.5: Microscale modeling of ferrogels. Effective material behavior of random monodisperse microstructures for load case II: (a) Magnetization $\bar{M}_{2}$ versus magnetic induction $\bar{B}$ and (b) mechanical stress $\bar{P}_{12}$ versus magnetic induction $\bar{B}$.

contrast, the decrease of the mechanical shear stress $\bar{P}_{12}$ with increasing magnetic induction indicates a negative magnetorheological effect for the unit cell with $30 \%$ particle volume fraction. Similar unsystematic behavior was found in the numerical studies of Kalina et al. [80] for three-dimensional unit cells with random monodisperse microstructures. In general, the magnetorheological effect is highly sensitive to morphological changes of the microstructure and strongly depends upon the material

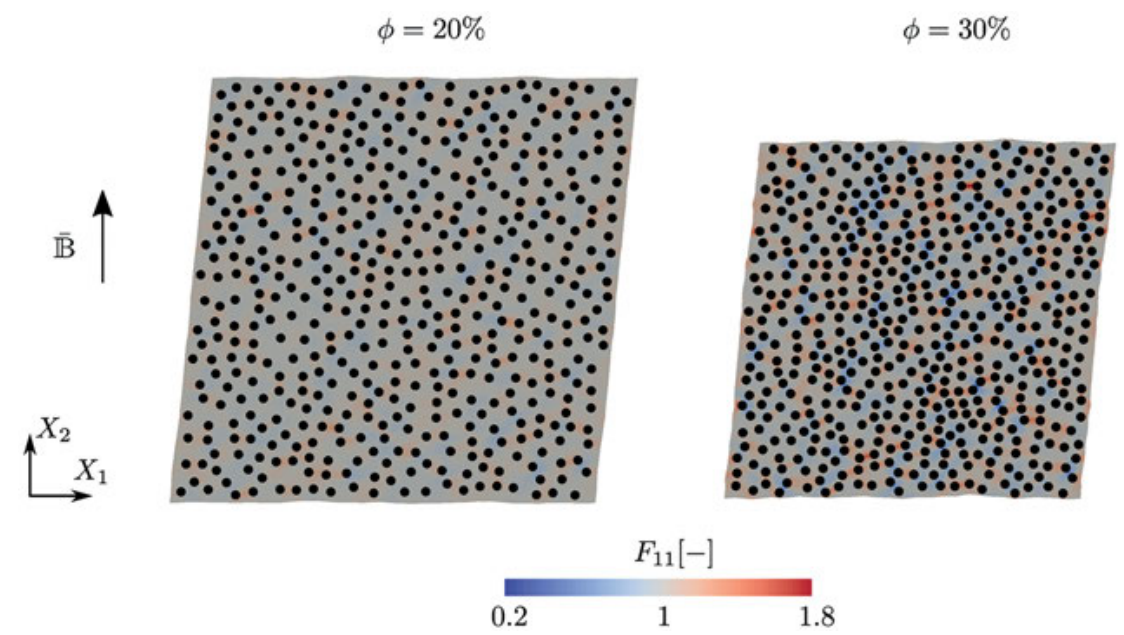

Figure 25.6: Microscale modeling of ferrogels. Spatial distribution of the local deformation gradient component $F_{11}$ in the unit cells with particle volume fraction $\phi=\{20 \%, 30 \%\}$ at a macroscopic magnetic induction of $\bar{B}=\bar{B} e_{2}$ with $\bar{B}=2 \mathrm{~T}$. 
behavior of the polymer matrix. A detailed analysis of this effect and its influencing factors is beyond the scope of the paper and a task for future investigations. Figure 25.6 shows the spatial distribution of the local deformation gradient component $F_{11}$ in the unit cells with a particle volume fraction of $\phi=\{20 \%, 30 \%\}$. Due to the fact that the particles try to rearrange themselves in energetically advantageous configurations, we observe particle movements in direction perpendicular to the applied magnetic field in order to form chain-like substructures. This leads to locally large deformations within the microstructure, especially at high particle volume fractions. In reality, this could cause debonding of the particles from the matrix material. From a numerical point of view, this can result in convergence issues triggered by mesh distortions.

\subsection{Conclusions}

The present work covers the multiphysics modeling of porous ferrogels at finite strains. Following the hierarchical structure of scales, we present theoretical and computational frameworks for two different modeling strategies: (i) the modeling of ferrogels at the macroscale level and (ii) the modeling of ferrogels at the microscale level. Prototypical constitutive models for locally nondissipative material behavior are derived in an enthalpy-based constitutive arrangement on the macroscale and an energy-based constitutive arrangement on the microscale. For both modeling approaches, a rigorous variational treatment is given, providing a canonically compact model structure. This consequently leads to a symmetric and compact formulation of the strongly coupled nonlinear multiphysics problem, which is ideally suited for an efficient finite element implementation. Representative boundary value problems outline the relevant features and capabilities of each modeling approach. The study on the macroscopic shape effect reveals that the field homogeneity is significantly higher in spheroidal shaped specimens with larger aspect ratios. Therefore, prolate spheroids are preferable in the experimental characterization of magnetoactive polymers. The proposed microscale model is embedded into a suitable energy-based scale transition scheme that allows to analyze the effective material response of the microstructure without the influence of any macrostructural effects. The study on the effective material behavior of random monodisperse microstructures for two load cases, which characterize the magnetostrictive and magnetorheological effect of the composite, reveals a qualitatively good agreement with experimental findings and theoretical studies in literature.

Future work will be devoted to the extension and parametrization of the constitutive model at the macroscale level based on a comprehensive data set generated from the microscale model. This approach allows the identification of an intrinsic material parameter set independent of macrostructural shape effects. From a numerical point of view, the development and implementation of a mesh update algorithm should be a future task since the mesh quality at large deformations has crucial influence on the convergence and stability of the finite element algorithm. 
Acknowledgments: The financial support of the German Research Foundation (DFG) through the Priority Programme (SPP) 1681, grant WA 2323/8, is gratefully acknowledged. The computations were performed on a High Performance Computing (HPC) system at the Center for Information Services and High Performance Computing (ZIH) at TU Dresden. The authors thank the ZIH for generous allocations of compute resources.

Author contribution: All the authors have accepted responsibility for the entire content of this submitted manuscript and approved submission.

Research funding: None declared.

Conflict of interest statement: The authors declare no conflicts of interest regarding this article.

\section{References}

1. Hu S-H, Liu T-Y, Liu D-M, Chen S-Y. Nano-ferrosponges for controlled drug release. J Contr Release 2007;121:181-9.

2. Zhao X, Kim J, Cezar CA, Huebsch N, Lee K, Bouhadir K, et al. Active scaffolds for on-demand drug and cell delivery. Proc Natl Acad Sci USA 2011;108:67-72.

3. Cezar CA, Kennedy SM, Mehta M, Weaver JC, Gu L, Vandenburgh H, et al. Biphasic ferrogels for triggered drug and cell delivery. Adv Healthcare Mater 2014;3:1869-76.

4. Li Y, Huang G, Zhang X, Li B, Chen Y, Lu T, et al. Magnetic hydrogels and their potential biomedical applications. Adv Funct Mater 2013;23:660-72.

5. Culver HR, Clegg JR, Peppas NA. Analyte-responsive hydrogels: intelligent materials for biosensing and drug delivery. Acc Chem Res 2017;50:170-8. PMID: 28170227.

6. Datta P. 17 - magnetic gels. In: Pal K, Banerjee I, editors Polymeric Gels, Woodhead Publishing Series in Biomaterials. Woodhead Publishing; 2018, pp. 441-65.

7. Hong S, Jung Y, Yen R, Chan HF, Leong KW, Truskey GA, et al. Magnetoactive sponges for dynamic control of microfluidic flow patterns in microphysiological systems. Lab Chip 2014;14:514-21.

8. Weeber R, Kantorovich S, Holm C. Deformation mechanisms in 2D magnetic gels studied by computer simulations. Soft Matter 2012;8:9923-32.

9. Tarama M, Cremer P, Borin DY, Odenbach S, Löwen H, Menzel AM. Tunable dynamic response of magnetic gels: impact of structural properties and magnetic fields. Phys Rev E 2014;90:042311.

10. Cremer P, Löwen H, Menzel AM. Tailoring superelasticity of soft magnetic materials. Appl Phys Lett 2015;107:171903.

11. Fischer L, Menzel AM. Magnetostriction in magnetic gels and elastomers as a function of the internal structure and particle distribution. J Chem Phys 2019;151:114906.

12. Biller AM, Stolbov OV, Raikher YL. Modeling of particle interactions in magnetorheological elastomers. J Appl Phys 2014;116:114904.

13. Biller AM, Stolbov OV, Raikher YL. Mesoscopic magnetomechanical hysteresis in a magnetorheological elastomer. Phys Rev E 2015;92:023202.

14. Menzel AM. Bridging from particle to macroscopic scales in uniaxial magnetic gels. J Chem Phys 2014;141:194907.

15. Pessot G, Weeber R, Holm C, Löwen H, Menzel AM. Towards a scale-bridging description of ferrogels and magnetic elastomers. J Phys Condens Matter 2015;27:325105. 
16. Galipeau E, Ponte Castañeda P. The effect of particle shape and distribution on the macroscopic behavior of magnetoelastic composites. Int I Solid Struct 2012;49:1-17.

17. Galipeau E, Ponte Castañeda P. A finite-strain constitutive model for magnetorheological elastomers: magnetic torques and fiber rotations. J Mech Phys Solid 2013;61:1065-90.

18. Javili A, Chatzigeorgiou G, Steinmann P. Computational homogenization in magneto-mechanics. Int J Solid Struct 2013;50:4197-216.

19. Kalina KA, Metsch P, Kästner M. Microscale modeling and simulation of magnetorheological elastomers at finite strains: a study on the influence of mechanical preloads. Int J Solid Struct 2016;102:286-96.

20. Kalina KA, Brummund J, Metsch P, Kästner M, Borin DY, Linke JM, et al. Modeling of magnetic hystereses in soft MRES filled with NDFEB particles. Smart Mater Struct 2017;26:105019-31.

21. Danas K. Effective response of classical, auxetic and chiral magnetoelastic materials by use of a new variational principle. J Mech Phys Solid 2017;105:25-53.

22. Metsch P, Kalina KA, Spieler C, Kästner M. A numerical study on magnetostrictive phenomena in magnetorheological elastomers. Comput Mater Sci 2016;124:364-74.

23. Metsch P, Kalina KA, Brummund J, Kästner M. Two- and three-dimensional modeling approaches in magneto-mechanics: a quantitative comparison. Arch Appl Mech 2018;89:47-62.

24. Landau LD, Bell J, Kearsley M, Pitaevskii L, Lifshitz E, Sykes J. Electrodynamics of continuous media. Elsevier, 2013, vol 8.

25. Truesdell C, Toupin R. The classical field theories. Berlin, Heidelberg: Springer Berlin Heidelberg; 1960. pp. 226-858.

26. Brown WF. Magnetoelastic interactions. Berlin: Springer, 1966, vol 9.

27. Tiersten HF. Coupled magnetomechanical equations for magnetically saturated insulators. J Math Phys 1964;5:1298-318.

28. Coleman BD, Dill EH. Thermodynamic restrictions on the constitutive equations of electromagnetic theory. Z Angew Math Phys 1971;22:691-702.

29. De Groot SR, Suttorp LG. Foundations of electrodynamics. Amsterdam: North-Holland Publishing Company; 1972.

30. Maugin GA, Eringen AC. On the equations of the electrodynamics of deformable bodies of finite extent. J Mécanique 1977;16:101-47.

31. Eringen AC, Maugin GA. Electrodynamics of continua I: foundations and solid media. New York: Springer-Verlag; 1990.

32. Pao Y-H. Electromagnetic forces in deformable continua. In: Nemat-Nasser S, editor, Mechanics today. Pergamon Press, 1978, vol 4, pp. 209-305.

33. Hutter K, Ven AA, Ursescu A. Electromagnetic field matter interactions in thermoelastic solids and viscous fluids. Springer-Verlag Berlin Heidelberg; 2006, vol 710.

34. Kovetz A. Electromagnetic theory. Oxford University Press; 2000.

35. Chatzigeorgiou G, Javili A, Steinmann P. Unified magnetomechanical homogenization framework with application to magnetorheological elastomers. Math Mech Solid 2014;19:193-211.

36. Keip M-A, Rambausek M. Computational and analytical investigations of shape effects in the experimental characterization of magnetorheological elastomers. Int J Solid Struct 2017;121: 1-20.

37. Keip M-A, Rambausek M. A multiscale approach to the computational characterization of magnetorheological elastomers. Int J Numer Methods Eng;107:338-60.

38. Rambausek M, Göküzüm FS, Nguyen LTK, Keip M-A. A two-scale FE-FFT approach to nonlinear magneto-elasticity. Int J Numer Methods Eng; 117:1117-42.

39. Nedjar B. A theory of finite strain magneto-poromechanics. J Mech Phys Solid 2015;84:293-312.

40. Attaran A, Brummund J, Wallmersperger T. Development of a continuum model for ferrogels. J Intell Mater Syst Struct 2017;28:1358-75. 
41. Attaran A, Brummund J, Wallmersperger T. Modeling and finite element simulation of the magnetomechanical behavior of ferrogels. J Magn Magn Mater 2017;431:188-91.

42. Gebhart $P$, Wallmersperger $T$. A general framework for the modeling of porous ferrogels at finite strains. J Mech Phys Solid 2019;122:69-83.

43. Kankanala S, Triantafyllidis N. On finitely strained magnetorheological elastomers. J Mech Phys Solid 2004;52:2869-908.

44. Ogden R, Steigmann D. Mechanics and electrodynamics of magneto- and electro-elastic materials. CISM International Centre for Mechanical Sciences. Wien: Springer-Verlag; 2011, 527.

45. Biot MA. General theory of three-dimensional consolidation. J Appl Phys 1941;12:155-64.

46. Biot MA. Theory of finite deformations of porous solids. Indiana Univ Math J 1972;21:597-620.

47. Lewis RW, Schrefler BA. The finite element method in the static and dynamic deformation and consolidation of porous media. John Wiley \& Sons; 1998.

48. Coussy O. Poromechanics. John Wiley \& Sons; 2004.

49. Coussy O. Mechanics and physics of porous solids. John Wiley \& Sons; 2010.

50. Coleman BD, Noll W. The thermodynamics of elastic materials with heat conduction and viscosity. Arch Ration Mech Anal 1963;13:167-78.

51. Ogden RW, Hill R. Large deformation isotropic elasticity: on the correlation of theory and experiment for compressible rubberlike solids. Proc R Soc Lond A Math Phys Sci 1972;328: 567-83.

52. Rice JR, Cleary MP. Some basic stress diffusion solutions for fluid-saturated elastic porous media with compressible constituents. Rev Geophys 1976;14:227-41.

53. Coussy O. Mechanics of porous continua. John Wiley \& Sons; 1995.

54. Gajo A, Denzer R. Finite element modelling of saturated porous media at finite strains under dynamic conditions with compressible constituents. Int J Numer Methods Eng 2011;85:1705-36.

55. Nedjar B. Formulation of a nonlinear porosity law for fully saturated porous media at finite strains. J Mech Phys Solid 2013;61:537-56.

56. Vuong A-T, Yoshihara L, Wall W. A general approach for modeling interacting flow through porous media under finite deformations. Comput Methods Appl Mech Eng 2015;283:1240-59.

57. Lefèvre V, Danas K, Lopez-Pamies 0 . Two families of explicit models constructed from a homogenization solution for the magnetoelastic response of MRES containing iron and ferrofluid particles. Int J Non Linear Mech 2020;119:103362.

58. Mukherjee D, Bodelot L, Danas K. Microstructurally-guided explicit continuum models for isotropic magnetorheological elastomers with iron particles. Int J Non Linear Mech 2020;120:103380.

59. Böger L, Nateghi A, Miehe C. A minimization principle for deformation-diffusion processes in polymeric hydrogels: constitutive modelling and Fe implementation. Int J Solid Struct 2017;121: $257-74$.

60. Teichtmeister S, Mauthe S, Miehe C. Aspects of finite element formulations for the coupled problem of poroelasticity based on a canonical minimization principle. Comput Mech 2019;64: 685-716.

61. Diguet G, Beaugnon E, Cavaillé J. Shape effect in the magnetostriction of ferromagnetic composite. J Magn Magn Mater 2010;322:3337-41.

62. Bodelot L, Voropaieff J-P, Pössinger T. Experimental investigation of the coupled magnetomechanical response in magnetorheological elastomers. Exp Mech 2018;58:207-21.

63. Raikher Y, Stolbov O. Magnetodeformational effect in ferrogel samples. J Magn Magn Mater 2003; 258-259:477-9.

64. Raikher YL, Stolbov OV. Deformation of an ellipsoidal ferrogel sample in a uniform magnetic field. J Appl Mech Tech Phys 2005;46:434-443.

65. Kalina KA, Metsch P, Brummund J, Kästner M. A macroscopic model for magnetorheological elastomers based on microscopic simulations. Int J Solid Struct 2020;193-194:200-12. 
66. Miehe C, Vallicotti D, Zäh D. Computational structural and material stability analysis in finite electro-elasto-statics of electro-active materials. Int J Numer Methods Eng;102:1605-37.

67. Ethiraj G, Miehe C. Multiplicative magneto-elasticity of magnetosensitive polymers incorporating micromechanically-based network kernels. Int J Eng Sci 2016;102:93-119.

68. Ciarlet PG. Mathematical elasticity: Volume I: three-dimensional elasticity. North-Holland; 1988.

69. Hill R. On constitutive macro-variables for heterogeneous solids at finite strain. Proc R Soc Lond A Math Phys Sci 1972;326:131-47.

70. Miehe C, Rosato D, Kiefer B. Variational principles in dissipative electro-magneto-mechanics: a framework for the macro-modeling of functional materials. Int J Numer Methods Eng;86: $1225-76$.

71. Semenov AS, Kessler H, Liskowsky A, Balke H. On a vector potential formulation for 3D electromechanical finite element analysis. Commun Numer Methods Eng 2006;22:357-75.

72. Vogel F, Bustamante R, Steinmann P. On some mixed variational principles in electroelastostatics. Int J Non Linear Mech 2012;47:341-54. Nonlinear Continuum Theories.

73. Vogel F, Bustamante R, Steinmann P. On some mixed variational principles in magnetoelastostatics. Int J Non Linear Mech 2013;51:157-69.

74. Miehe C, Vallicotti D, Teichtmeister S. Homogenization and multiscale stability analysis in finite magneto-electro-elasticity. application to soft matter ee, me and mee composites. Comput Methods Appl Mech Eng 2016;300:294-346.

75. Bustamante R, Dorfmann A, Ogden R. On variational formulations in nonlinear magnetoelastostatics. Math Mech Solid 2008;13:725-45.

76. Šilhavý M. A variational approach to nonlinear electro-magneto-elasticity: convexity conditions and existence theorems. Math Mech Solid 2018;23:907-28.

77. Gollwitzer C, Turanov A, Krekhova M, Lattermann G, Rehberg I, Richter R. Measuring the deformation of a ferrogel sphere in a homogeneous magnetic field. J Chem Phys 2008;128:164709.

78. Filipcsei G, Zrínyi M. Magnetodeformation effects and the swelling of ferrogels in a uniform magnetic field. J Phys Condens Matter 2010;22:276001.

79. Jolly MR, Carlson JD, Muñoz BC, Bullions TA. The magnetoviscoelastic response of elastomer composites consisting of ferrous particles embedded in a polymer matrix. J Intell Mater Syst Struct 1996;7:613-22.

80. Kalina KA, Raßloff A, Wollner M, Metsch P, Brummund J, Kästner M. Multiscale modeling and simulation of magneto-active elastomers based on experimental data. Phys Sci Rev 2020. 
